\title{
Stage-Oriented CT Classification and Intermodal Evolution Model in Hepatic Alveolar Echinococcosis
}

\section{Stadienorientierte CT-Klassifikation und intermodales Evolutionsmodell bei hepatischer alveolärer Echinokokkose}

\section{(c) (i) $(9)$}

\section{Authors}

Tilmann Graeter ${ }^{1}$, Julian Schmidberger ${ }^{2}$

Affiliations

1 Department of Diagnostic and Interventional Radiology, Universitätsklinikum Ulm, Germany

2 Department of Internal Medicine I, Universitätsklinikum Ulm, Germany

Key words

computed tomography, intermodal classification, lesion evolution, alveolar echinococcosis

received 25.06 .2021

accepted 14.10.2021

published online 26.01 .2022

Bibliography

Fortschr Röntgenstr 2022; 194: 532-544

DOI 10.1055/a-1710-3669

ISSN 1438-9029

(c) 2022. The Author(s).

This is an open access article published by Thieme under the terms of the Creative Commons Attribution-NonDerivative-NonCommercial License, permitting copying and reproduction so long as the original work is given appropriate credit. Contents may not be used for commecial purposes, or adapted, remixed, transformed or built upon. (https://creativecommons.org/licenses/by-nc-nd/4.0/)

Georg Thieme Verlag KG, Rüdigerstraße 14,

70469 Stuttgart, Germany

Correspondence

Herr Prof. Dr. Tilmann Graeter

Department of Diagnostic and Interventional Radiology,

University Hospital Ulm, Albert-Einstein-Allee 23, 89081 Ulm, Germany

Tel.: +49/7 31/50061175

tilmanngraeter@gmx.de

\section{ABSTRACT}

Background Alveolar echinococcosis (AE) is one of the most dangerous human parasitoses. The main site of disease manifestation is the liver (about $98 \%$ ). The Echinococcus Multilocularis Ulm Classification for Computed Tomography (EMUCCT), presented in 2016, was the first compilation of CT morphological criteria of hepatic AE. Studies based on EMUC-CT made it possible to draw conclusions about the development of the lesions in the course of disease beyond purely diagnos- tic typing. Among the most important findings of these precursor studies was that EMUC-CT type IV presented as an initial lesion, whereas EMUC-CT type III lesions were mostly associated with an advanced disease constellation. An intermodal view of image morphological criteria provides further multi-layered indications for lesion evolution.

Method With the "Alveolar Echinococcosis Ulm Classification" (AEUC), a revision of the previous EMUC-CT was carried out with stage-oriented reorganization of the primary morphologies. Furthermore, an intermodal classification scheme for the evolution of hepatic AE lesions based on AEUC, MRI Kodama classification, and aspects of ultrasound could be outlined.

Results The first stage-oriented CT classification of hepatic AE "AEUC" is based with respect to its lesion characterization on the separate consideration of two classification pillars, the five "primary morphologies", AEUC I-V (AEUC II-IV with subcriteria) and the five "patterns of calcification". In addition, an intermodal classification scheme presents five stages of lesion evolution: "initial stage", "progressive stage", "advanced stage", "transitional stage" and "regressive stage".

Conclusion The imaging modalities differ with respect to their visualization of lesion criteria. This underlines the need for unimodal classification systems. Staging of an AE lesion can be done more accurately by evaluating different modalities.

Key Points:

- The AEUC provides a stage-oriented CT classification for hepatic AE.

- Aspects of different modalities allow a more multi-layered view of lesion evolution.

- More accurate staging can be achieved by combining different modalities.

\section{Citation Format}

- Graeter T, Schmidberger J. Stage-Oriented CT Classification and Intermodal Evolution Model in Hepatic Alveolar Echinococcosis. Fortschr Röntgenstr 2022; 194: 532-544 


\section{ZUSAMMENFASSUNG}

Hintergrund Die alveoläre Echinokokkose (AE) ist eine der gefährlichsten humanen Parasitosen. Hauptmanifestationsort der Erkrankung ist mit etwa 98\% die Leber. Die 2016 vorgestellte „Echinococcus Multilocularis Ulm Classification for Computed Tomography“ (EMUC-CT) war die erste Zusammenstellung CT-morphologischer Kriterien der hepatischen AE. Studien auf Basis der EMUC-CT ermöglichten es, über die rein diagnostische Typisierung hinaus Rückschlüsse auf die Entwicklung der Läsionen im Krankheitsverlauf zu ziehen. Zu den wichtigsten Erkenntnissen dieser Vorläuferstudien gehörte, dass sich Typ IV der EMUC-CT als Initialläsion präsentierte, während EMUC-CT Typ III-Läsionen meist mit einer fortgeschrittenen Krankheitskonstellation verbunden waren. Eine intermodale Betrachtung bildmorphologischer Kriterien gibt weitere Hinweise für eine Läsionsevolution.

Methode Mit der „Alveolar Echinococcosis Ulm Classification“ (AEUC) erfolgte eine Revision der bisherigen EMUC-CT mit einer stadienorientierten Neuordnung der Primärmor- phologien. Zudem konnte ein intermodales Klassifikationsschema zur Evolution hepatischer AE-Läsionen anhand der AEUC, der MRT Kodama-Klassifikation und Aspekten des Ultraschalls skizziert werden.

Ergebnisse Die erste stadienorientierte CT-Klassifikation hepatischer AE „AEUC“ basiert in ihrer Läsionscharakterisierung auf der getrennten Betrachtung zweier Klassifikationssäulen, den 5 „Primärmorphologien“, AEUC I-V (AEUC II-IV mit Subkriterien), und den 5 „Kalzifikationsmustern“. Darüber hinaus präsentiert ein intermodales Klassifikationsschema 5 Stadien der Läsionsevolution: „Initialstadium“, „Progressionsstadium“, „fortgeschrittenes Stadium“, „Übergangsstadium“ und „Regressionsstadium“.

Schlussfolgerung Die bildgebenden Modalitäten unterscheiden sich in der Darstellung von Läsionskriterien. Dies unterstreicht die Notwendigkeit unimodaler Klassifikationssysteme. Die Stadienzuordnung einer AE-Läsion kann unter Wertung verschiedener Modalitäten genauer erfolgen.

\section{Introduction}

Alveolar echinococcosis (AE), which is caused by the ingestion of eggs of the small fox tapeworm Echinococcus multilocularis, is the most dangerous human parasitosis if left untreated [1, 2]. Cases of $\mathrm{AE}$ are seen in the northern hemisphere with the main endemic regions being in central Europe and western China. BadenWürttemberg and Bavaria are the main endemic regions in Germany with a tendency to spread to other parts of the territory [3-8]. In the period 1992-2016, the prevalence of AE in Germany was $0.64 / 100000$ inhabitants, with a prevalence of 2.18/100 000 inhabitants in Baden-Württemberg and 1.48/ 100000 in Bavaria [3-8]. The greatest number of cases of the disease worldwide have been reported in China. The main risk factors include the keeping of dogs, participation in agricultural or forestry activities, and the consumption of plants from the garden [9]. Human beings are dead-end hosts in the development cycle of this parasitic disease. The liver is the main site of manifestation of the disease with its involvement in approximately $98 \%$ of cases. All other organs can be affected, albeit much more rarely [10, 11]. In central Europe, curative surgical therapy is still possible in one third of cases with the safe distances of the resection margins having been reevaluated in recent years [12]. In the other cases, typically lifelong parasitostatic treatment with benzimidazole is implemented with a good prognosis and usually normal life expectancy. Hepatobiliary complications as can occur in advanced disease can be managed primarily with an intervention and medication [13]. The WHO case definition according to Brunetti et al. defines the categories "possible", "probable", and "confirmed" for diagnosis based on serology, imaging, and histology. Positive serology alone does not determine disease. The category "confirmed" requires histological confirmation. Imaging with ultrasound (US), magnetic resonance imaging (MRI), and computed tomography (CT) often combined with positron emission tomography (PET) is an important part of the diagnosis of $A E$ in this context [14]. PET-CT is an important parameter in the evaluation of the disease both initially and over the course of the disease. It must be taken into consideration that $A E$ lesions are not perfused and the PET activity in the marginal rim only provides information about inflammatory activity in the surrounding tissue [15]. Negative PET findings do not necessarily indicate devitalized lesions, e. g., in immunosuppressed patients. It must also be taken into consideration that inflammatory activity can initially increase at the start of drug therapy [16].

Intermodal imaging of complex lesions is helpful because, for example, lesion margins (also for possible surgical planning), small lesions, and calcifications are better visualized with $\mathrm{CT}$, while alveoli are better visualized with MRI. US, which is usually a primary diagnostic method in the case of the detection of incidental findings, offers the best resolution of the internal structures of small lesions and using ultrasound contrast-agent has advantages regarding differential diagnosis showing typical absence of contrast enhancement in $\mathrm{AE}$ lesions.

Since AE lesions have a polymorphic appearance on imaging and can mimic various benign and malignant liver masses, diagnosis of this rare parasitic disease remains difficult. Therefore, $A E$ imaging studies are important. To date, there are three morphological classifications for the different imaging modalities for hepatic AE. The Kodama classification from 2003 defines five types of AE lesions on MRI, while the "Echinococcus Multilocularis Ulm Classification for Ultrasound" (EMUC-US) was introduced as a descriptive diagnostic instrument for US in 2015 [17, 18]. The EMUC-US differentiates between "hailstorm pattern", "pseudocystic pattern", "ossification pattern”, "hemangioma-like pattern", and "metastasis-like pattern". However, US diagnosis is difficult in the case of complex, poorly definable AE lesions and significant calcification. The T2w-based Kodama classification for MRI, which is discussed in greater detail in the subsequent 


\section{Kodama Classification Type - Description}
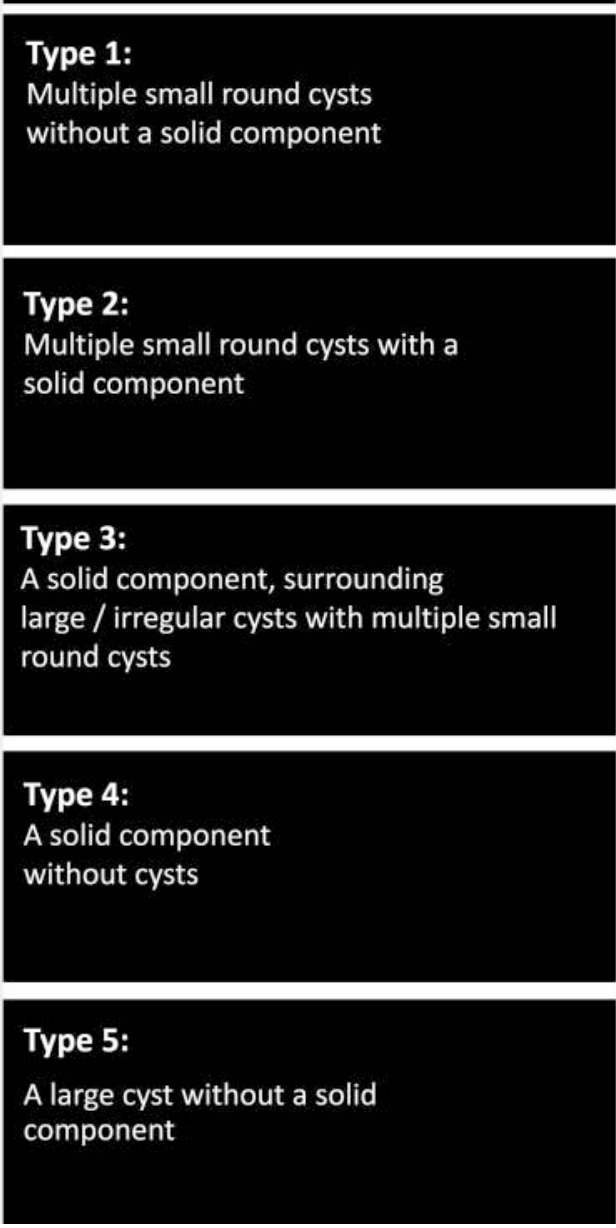
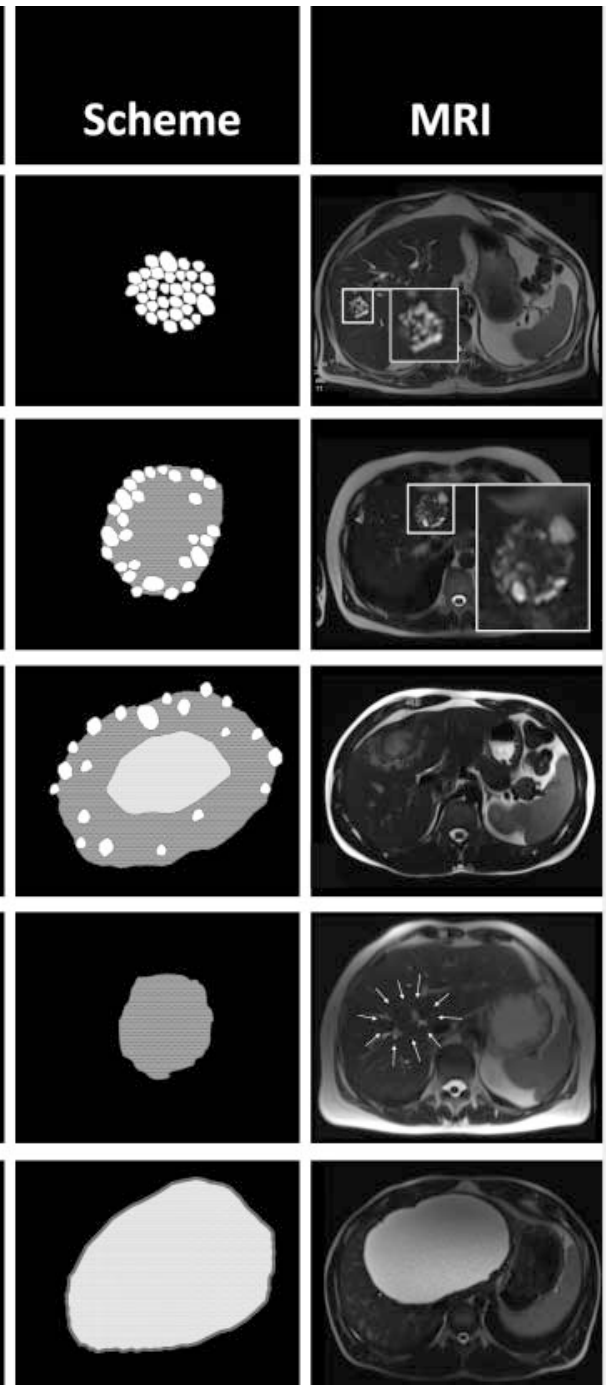

D Fig. 1 Scheme of T2w-based Kodama classification and MRI sample images of the different lesion types.

intermodal evaluation, is shown in $\mathbf{F i g . 1}$. The "Echinococcus Multilocularis Ulm Classification for Computed Tomography" (EMUC-CT), which was introduced in 2016, provided a structured compilation of the most important morphological CT criteria with five primary morphological types and typical calcification patterns to improve the diagnosis of AE [19].

There is a stage-adapted intermodal classification based on the image morphology of the liver lesions for cystic echinococcosis (CE) but not for AE [14]. Such a classification is more difficult for $\mathrm{AE}$ due to the more complex lesions. In addition, differences in image information between imaging modalities plays a greater role in $\mathrm{AE}$ than in CE. Therefore, the existing, not yet stage-adapted AE classifications for MRI, US, and CT are not always directly comparable [20]. In contrast to the presented primary diagnostic classifications of hepatic AE for the individual modalities, the PNM classification, which was introduced in 2006, allows diagnosis of intrahepatic and extrahepatic spread of AE [21].

The most recent study results indicate more than the purely diagnostic value of the morphological typing of hepatic $A E$ and allow conclusions about the evolution of lesions over the course of the disease.

Therefore, on the basis of the EMUC-CT, the lesion types were examined regarding their behavior under benzimidazole treatment, their prevalence in various endemic regions with different degrees of disease progression, their correlation with intrahepatic manifestation criteria (size, vascular/biliary infiltration) and simultaneous extrahepatic involvement, the density values of their cystoid components, their PET activity and serology, and finally regarding histopathological criteria [22-27].

Important information provided by these studies was that type IV of the EMUC-CT represents an initial lesion, while EMUC-CT type III is associated with an advanced disease constellation [23, 24].

The frequent absence of PET activity in small EMUC-CT type IV lesions can be explained histologically by centralization of the alveolus, which therefore often is still surrounded by a margin of isolating solid necroses, while other lesion types with a larger size represent various stages of more advanced $A E$ lesions in which the inflammatory activity is again located at the margin within the scope of an exacerbation [26]. 


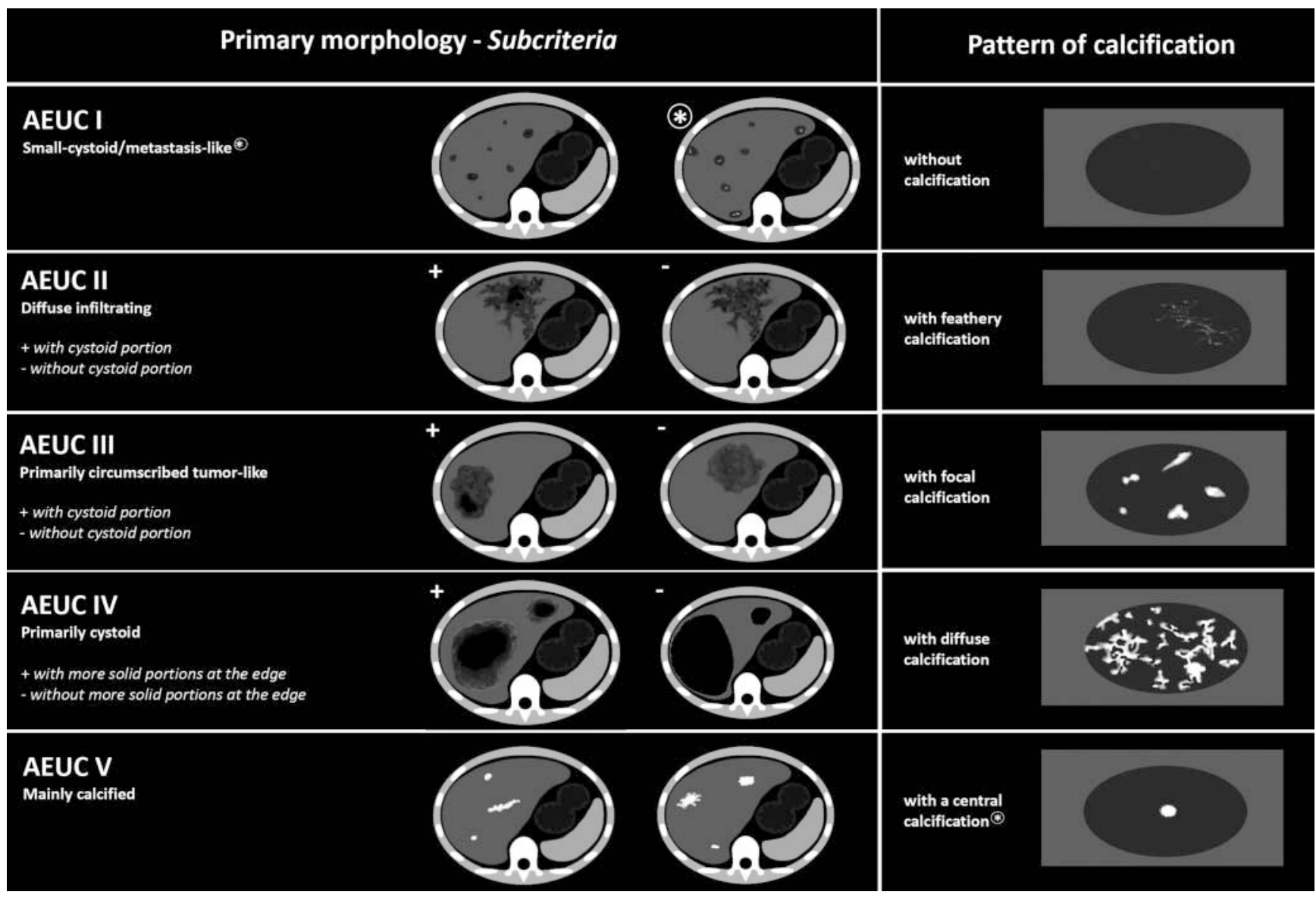

- Fig. 2 Overview of the new CT classification AEUC. Left: "Primary morphology" AEUC I-V and their subcriteria - applicable to AEUC II, III, and IV (+/-). Right: "Patterns of calcification" within a lesion; AEUC = Alveolar Echinococcosis Ulm Classification. The two pillars of the classification are primarily considered separately and can then in principle be freely combined. There are two exceptions: The pattern of calcification "with a central calcification*" can occur only with primary morphology AEUCI*, and primary morphology AEUC $V$ is not further characterized by a pattern of calcification.

A histopathological/CT morphological comparison study based on histological criteria provided additional important information about the development status of AE lesion types in the course of the disease thus suggesting lesion evolution. As a result of this study, type IV according to EMUC-CT was defined as the "initial stage", types I and II as the "progressive stage", type III as the "advanced stage" and type $V$ as the "regressive stage" [27].

In summary the precursor studies indicate stage-based evolution of hepatic AE lesions, with lesion progression of the primary morphology being able to be prevented by parasitostatic treatment with an increase in calcification [22-27].

Therefore, a revision of the EMUC-CT with reclassification of the primary morphological lesion types based on aspects of lesion evolution was considered helpful since the EMUC-CT only numbered the primary morphologies based on the frequency of occurrence within the original German collective without consideration of possible development stages [19]. Accordingly, the "Alveolar Echinococcosis Ulm Classification" (AEUC) provides a new, stageoriented CT classification of hepatic AE.

Finally, based on the results of the most recent AE studies, an intermodal classification model of the evolution of hepatic AE lesions with five stages can be outlined, taking into consideration the AEUC for CT, the MRI Kodama classification, and aspects of US.

\section{Main text}

\section{Basis of the revision of the CT classification}

Transformation of the EMUC-CT into the stage-oriented AEUC

The EMUC-CT classification was renamed AEUC to avoid confusion with the previous typing as well as possible parallels to the persisting EMUC-US classification since the lesion types cannot always be readily transferred between $C T$ and US because of differences in the methods and because of possible calcification [18-20]. A further reason for the renaming of the $C T$ classification was that the disease being classified is alveolar echinococcosis and it seemed more logical to use the name of the disease and not that of the parasite, Echinococcus multilocularis, in the nomenclature.

Like the EMUC-CT, the AEUC also uses two independent complementary pillars, namely primary morphology and calcification pattern ( $\triangleright$ Fig. 2), to characterize AE lesions [19, 22, 26].

The avoid confusion between the AEUC typology and the typology of the former EMUC-CT classification system, the new primary morphologies were defined as "AEUC I-V" and no longer as "type I-V" [19]. The descriptive names of the primary morphologies already presented in the EMUC-CT were maintained in the 
- Table 1 Criteria for primary morphologies AEUC I-V including sub-criteria for AEUC II-IV (left column) and for AEUC patterns of calcification (right column) - compare $>$ Fig. $\mathbf{2 , ~} 3$

Primary morphology - subcriteria

AEUC I: "Small cystoid/metastasis-like*"

(no additional subcriteria defined)

- Small hypodense round or oval lesions

- Appearance results from a single microcyst or a central microcyst/few microcysts with a surrounding rim of solid necrosis ( $\triangleright$ Fig. $3 a-d)$.

- A central calcification*, can only occur in AEUCI* ( Fig. 3c, d).

- In the absence of a central calcification ( $\triangleright$ Fig. 3a, b), similarity with small, normal cyst, protein-rich cyst, or hypodense metastasis

\section{AEUC II: “Diffuse infiltrating”}

(additional subcriteria have to be defined - see below)

- Often no defined central focus of growth ( $\mathbf{F i g}$. $3 \mathbf{e}-\mathbf{h}$ )

- Diffuse, at times fan-shaped, sometimes long offshoots

- Often unsharp border ( $>$ Fig. $3 \mathbf{e}, \mathbf{g}$ ).

- If border is better defined, no convex shape (like AEUC III), but with invasive longitudinal or concave margin ( $\vee$ Fig. $\mathbf{3 f}$ - the example also shows a congested bile duct)

Subcriteria - with/without cystoid portion:

Larger cystoid structures (liquid necrosis) within the lesion, either central or decentralized ( $\triangleright$ Fig. 3e, f).

\section{AEUC III: "Primarily circumscribed tumor-like"}

(additional subcriteria have to be defined - see below)

- Circumscribed with defined center of lesion ( $\mathbf{F i g} \cdot \mathbf{3 i}-\mathbf{I}$ )

- Convex shape

- No or just short offshoots

Subcriteria - with/without cystoid portion:

AEUC III lesions may (as AEUC II) also contain larger cystoid portions (liquid necrosis) that may be located centrally ( $\triangleright$ Fig. $\mathbf{3 i}$ ) or (distinct from AEUC IV) may be decentralized ( $\triangleright$ Fig. $\mathbf{3 j}$ ).

\section{AEUC IV "Primarily cystoid"}

(additional subcriteria have to be defined - see below)

- Round or oval with intermediate $(\triangleright$ Fig. $\mathbf{3}$ m, o) or huge size ( $\vee$ Fig. $\mathbf{3 n}, \mathbf{p})$

- Quite clear, sometimes irregular margin

- Dominant cystoid component (liquid necrosis) ( $>$ Fig. 3m-p)

Subcriteria - with/without more solid portions at the edge:

AEUC IV may present as purely cystoid or show a more solid marginal rim of varying thickness, while the cystoid basic structure remains relatively central ( $\mathbf{F i g . ~} 3 \mathrm{~m}, \mathbf{n}$ ).

\section{AEUC V, "Predominantly calcified"}

(no additional subcriteria defined)

- Predominantly calcified component ( $>$ Fig. $\mathbf{3 q - t}$ )

- Occasionally very small residual amounts of solid necrosis

- Rather small or medium sized

- Sole primary morphology without further, separate definition of a pattern of calcification for the entire lesion description

\section{Patterns of calcification}

\section{"Without calcification"}

no calcification in a lesion

\section{"With feathery calcification"}

Delicate, foggy, or cobweb-like calcifica-

tion of varying degrees

\section{"With focal calcification"}

Distributed, point- or comma-shaped calcification
"With diffuse calcification"

confluent calcification with different morphological characteristics new classification in accordance with the changed order as specified in the following.

In contrast to the EMUC-CT, stage-oriented classification of the primary morphologies was used in the AEUC. Accordingly, the former EMUC-CT type IV as the initial small lesion is the first morphology of the new classification scheme (AEUC I "small cystoid/metastasis-like”). The other primary morphologies representing progressive and advanced lesions were then able to be arranged in ascending order so that EMUC-CT type I was changed to AEUC II "diffuse infiltrating", EMUC-CT type II to AEUC III "primarily circumscribed tumor-like", and EMUC-CT type III to AEUC IV "primarily cystoid". EMUC-CT type $V$ was able to be maintained as a possible regressive stage in the new classification as AEUC V "primarily calcified” [27]. In contrast to EMUC-CT, the primary morphology "primarily cystoid" was not divided into the size-dependent subgroups $a / b$ in the AEUC since precursor studies found no advantage to such a differentiation [26, 27]. The subcriteria introduced in the EMUC-CT to be selected for the primary morphologies "diffuse infiltrating", "primarily circumscribed tumor-like", and "primarily cystoid" were retained in the AEUC in order to provide a more precise description of the lesion morphology [19].

The possible calcification patterns were reduced from six in the EMUC-CT to five in the AEUC since the relatively nonspecific and purely spatial pattern "with calcification primarily at the edge" was eliminated. However, the special pattern "with a central calcification*" was retained. Occurrence of this calcification pattern with the typical central point-shaped manifestation as a possible 


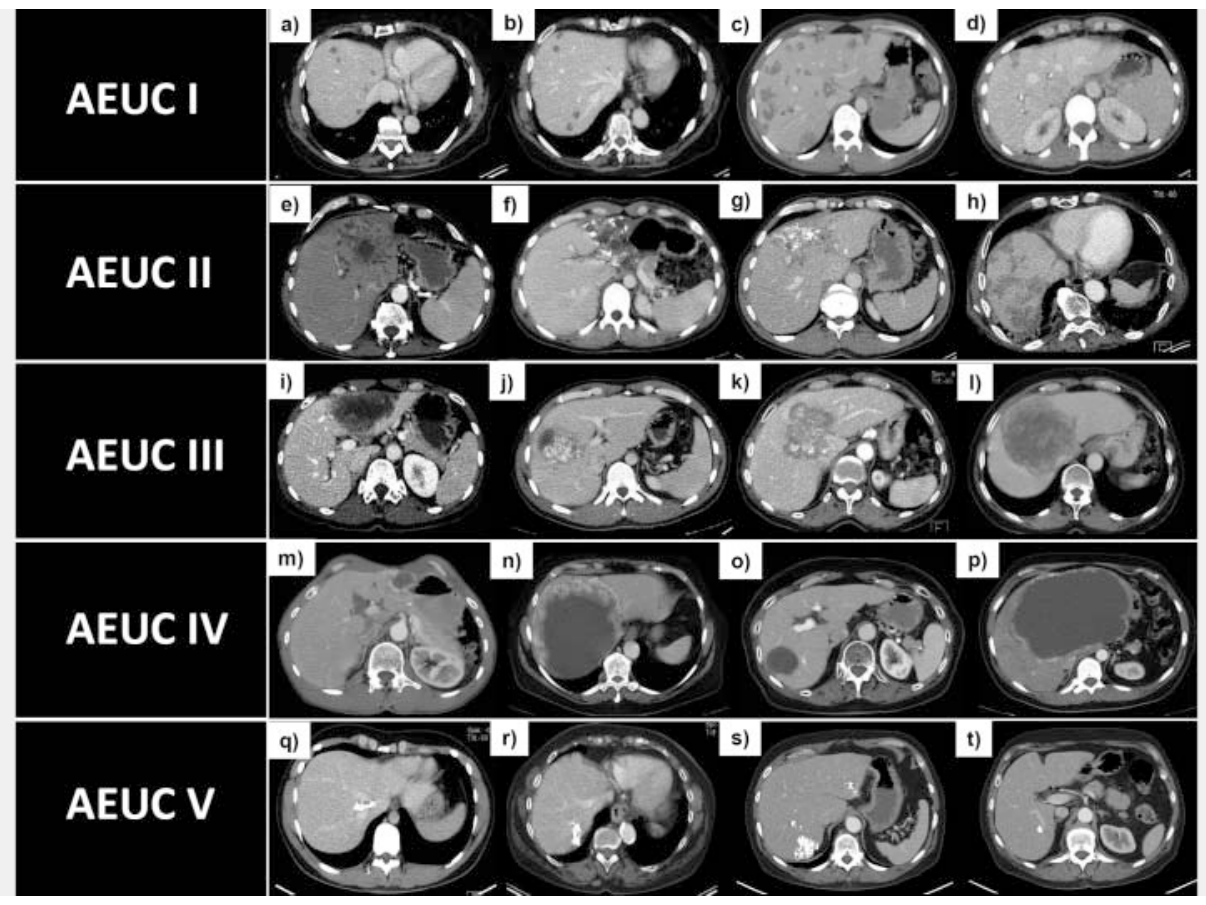

- Fig. 3 Sample CT image of the different primary morphologies AEUC I-V (without focus on calcification patterns). AEUC I a-d, AEUC II e-h, AEUC III i-I, AEUC IV $\mathbf{m}-\mathbf{p}$, AEUC V q-t. The subcriteria for AEUC II-IV are shown in two sample images each on the left and right.

pathognomonic indicator is still possible only in the primary morphology "small cystoid/metastasis-like*" and is therefore marked with an asterisk like this primary morphology [19, 23, 24, 26]. Besides the special nature of a central calcification, it should be mentioned that calcifications can increase over the course of the disease or during treatment. In particular, feathery calcifications are on the other hand associated with the highest inflammatory activity of vital lesions $[22,26,28]$. The further calcification patterns were therefore adopted in the AEUC.

The remaining five calcification patterns of the AEUC are: "Without calcification", "with feathery calcification", "with focal calcification", "with diffuse calcification", and "with a central calcification*".

\section{Representation and application of the AEUC}

Primary morphologies and calcification patterns

The criteria for the primary morphologies AEUC I-V and for the AEUC calcification patterns are listed in $>$ Table 1. Refer to the left side of $>$ Fig. 2 (schematic) and $>$ Fig. 3 (CT images) for the primary morphologies. The calcification patterns are shown schematically on the right side of $\mathbf{r i g} \mathbf{~} \mathbf{2}$.

As in the EMUC-CT, the two classification pillars must also be combined in the AEUC for a comprehensive description of the lesion with the primary morphology and the corresponding subcriterion for AEUC II-IV first followed by the calcification pattern. In contrast to the other primary morphologies, the primary morphology "primarily calcified" (AEUC V) is the only one not further defined within the AEUC by a supplementary calcification pattern due to the already dominant calcification [19] ( Fig. 2).

\section{Reporting of hepatic AE in CT}

For detailed evaluation of hepatic AE on CT, a structured reporting form was developed on the basis of the AEUC ( $\triangleright$ Fig. 4). In addition to AEUC classification, the form can also be used to document additional criteria of hepatic AE manifestation that were identified as important with respect to the overall assessment at the onset and over the course of the disease [19, 22-28].

Initial use of the AEUC in a patient collective of the national AE database

The AEUC was applied empirically in a collective of $n=140 \mathrm{AE}$ patients of the University Hospital Ulm. The assessment was performed independently by a radiologist with a number of years of experience in the field of $\mathrm{AE}$ as well as by a fourth-year radiology resident with good knowledge of $C T$ imaging. There was an interrater reliability (Cohen's Kappa) in relation to the primary morphologies of 0.8268 (95\% Cl: $0.7453-0.9084$ ) with $p<0.0001$. The analysis of the not yet published data from the collective is provided in $>$ Table 2 .

\section{Intermodal classification model of lesion evolution in hepatic AE}

An intermodal classification model of hepatic AE lesions, which takes the primary morphologies of the AEUC for CT, the MRI Kodama classification as well as aspects of US into consideration, can be outlined in the following based on recent study results. The evolution of the lesion morphologies is presented in consideration of the most important intermodal aspects ( $\triangleright$ Fig. 5a) [17, 22-27, 29-31]: 


\section{Registration form}

\section{Alveolar Echinococcosis Ulm Classification (AEUC)}

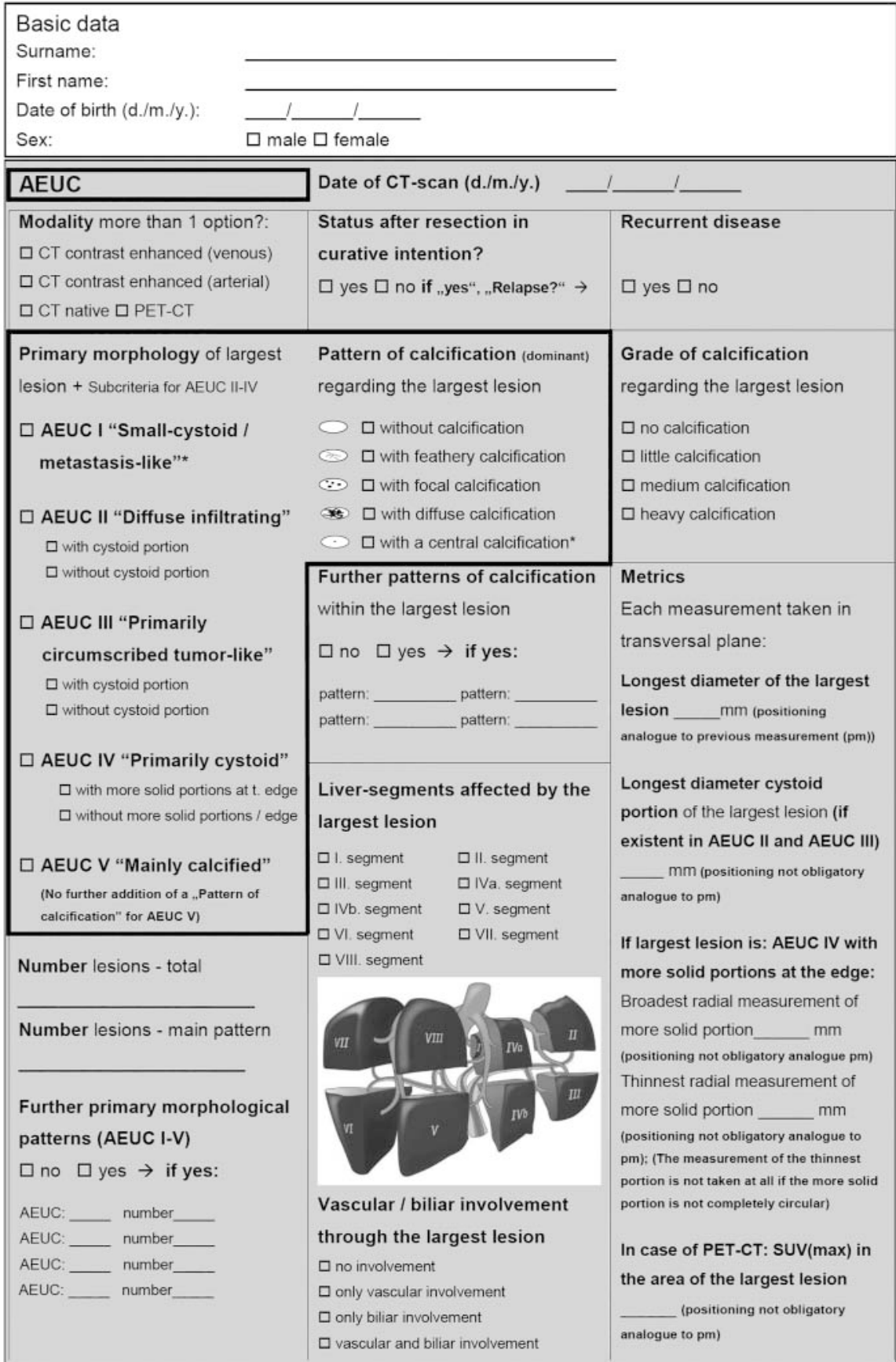

- Fig. 4 Structured reporting form based on the AEUC. The thick-framed area of the recording sheet marks the actual AEUC. In addition to classification according to AEUC, the recording form also allows documentation of other important criteria of hepatic AE manifestation with regard to the overall assessment at baseline and during the course. Furthermore, if there is more than one liver lesion, the total number of lesions and possible further primary morphologies of smaller lesions can be noted with the number of their respective occurrence. 
- Table 2 Basic data of the study collective and evaluation of the largest hepatic AE lesion in each case according to AEUC ( $n=140)$.

\begin{tabular}{|c|c|c|c|}
\hline$N=140$ & $\mathrm{~N}(\%)$ & Mean \pm SD (median) & (Min-max) \\
\hline Age (years) & & $60.44 \pm 15.90(63.00)$ & $21.00-88.00$ \\
\hline \multicolumn{4}{|l|}{ Gender } \\
\hline Male & $59(42.14 \%)$ & & \\
\hline Female & $81(57.86 \%)$ & & \\
\hline \multicolumn{4}{|l|}{ Primary morphology } \\
\hline AEUCI & $34(24.29 \%)$ & & \\
\hline AEUC II & $50(35.71 \%)$ & & \\
\hline With cystoid portion & $15(30.00 \%)$ & & \\
\hline Without cystoid portion & $35(70.00 \%)$ & & \\
\hline AEUC III & $36(25.71 \%)$ & & \\
\hline With cystoid portion & $8(22.22 \%)$ & & \\
\hline Without cystoid portion & $28(77.78 \%)$ & & \\
\hline AEUC IV & $16(11.43 \%)$ & & \\
\hline With more solid portions at the edge & $9(56.25 \%)$ & & \\
\hline Without more solid portions at the edge & $7(43.75 \%)$ & & \\
\hline AEUC V & $4(2.86 \%)$ & & \\
\hline \multicolumn{4}{|l|}{ Pattern of calcification } \\
\hline Without calcification & $44(31.43 \%)$ & & \\
\hline With feathery calcification & $17(12.14 \%)$ & & \\
\hline With focal calcification & $21(15.00 \%)$ & & \\
\hline With diffuse calcification & $46(32.86 \%)$ & & \\
\hline With a central calcification & $8(5.71 \%)$ & & \\
\hline No assignment of calcification for AEUC $V$ & $4(2.86 \%)$ & & \\
\hline Mean lesion size (mm) & & $62.43 \pm 41.07(52.00)$ & $8.00-195.00$ \\
\hline \multicolumn{4}{|l|}{ AEUC lesion sizes (mm) } \\
\hline AEUCI & & $20.50 \pm 8.84(20.00)$ & $8.00-41.00$ \\
\hline AEUC II & & $77.88 \pm 35.77(76.00)$ & $30.00-183.00$ \\
\hline AEUC III & & $62.22 \pm 26.06(56.00)$ & $29.00-129.00$ \\
\hline AEUC IV & & $111.13 \pm 45.97(105.50)$ & $47.00-195.00$ \\
\hline AEUC V & & $32.75 \pm 13.15(31.50)$ & $18.00-50.00$ \\
\hline
\end{tabular}

First of all, according to the aforementioned histopathological/ CT-morphological comparison study based on the EMUC-CT, the reorganized AEUC can be underpinned by the stages of lesion evolution described there: AEUCI "small cystoid/metastasis-like" corresponds to the "initial stage", AEUC II "diffuse infiltrating" and AEUC III "primarily circumscribed tumor-like" correspond to the "progressive stage", AEUC IV "primarily cystoid" corresponds to the "advanced stage" and AEUC V "primarily calcified" corresponds to lesions in the "regressive stage" [27].

Initial "small cystoid/metastasis-like" lesions that arise from a single early alveolus can either progress with the formation of additional alveoli or die off - typically as a result of progressive centrifugal calcification or due to increasing solid necrosis that consumes the central alveolus [26]. Temporary hibernation of such lesions with potential for reactivation can also occur. The histological lesion structure includes typical centralization of the active alveolus and the lamellar body surrounded by isolating solid necrosis which consequently prevents an inflammatory reaction in the surrounding tissue on PET $[25,26]$. For correct histological diagnosis, the center of such lesions must therefore be punctured so that the lamellar body is included [27].

A sample case in which MRI was originally used for a clinical reason other than diagnosing $A E$ incidentally shows directly traceable initial AE lesion evolution ( $\mathbf{F i g . 6}$ ). Therefore, such small initial lesions can also develop into larger, progressive lesions within a relatively short time period, probably when the body is not able to form or maintain the isolating necrosis around the vital alveolus. In the present case disease progression was initially 


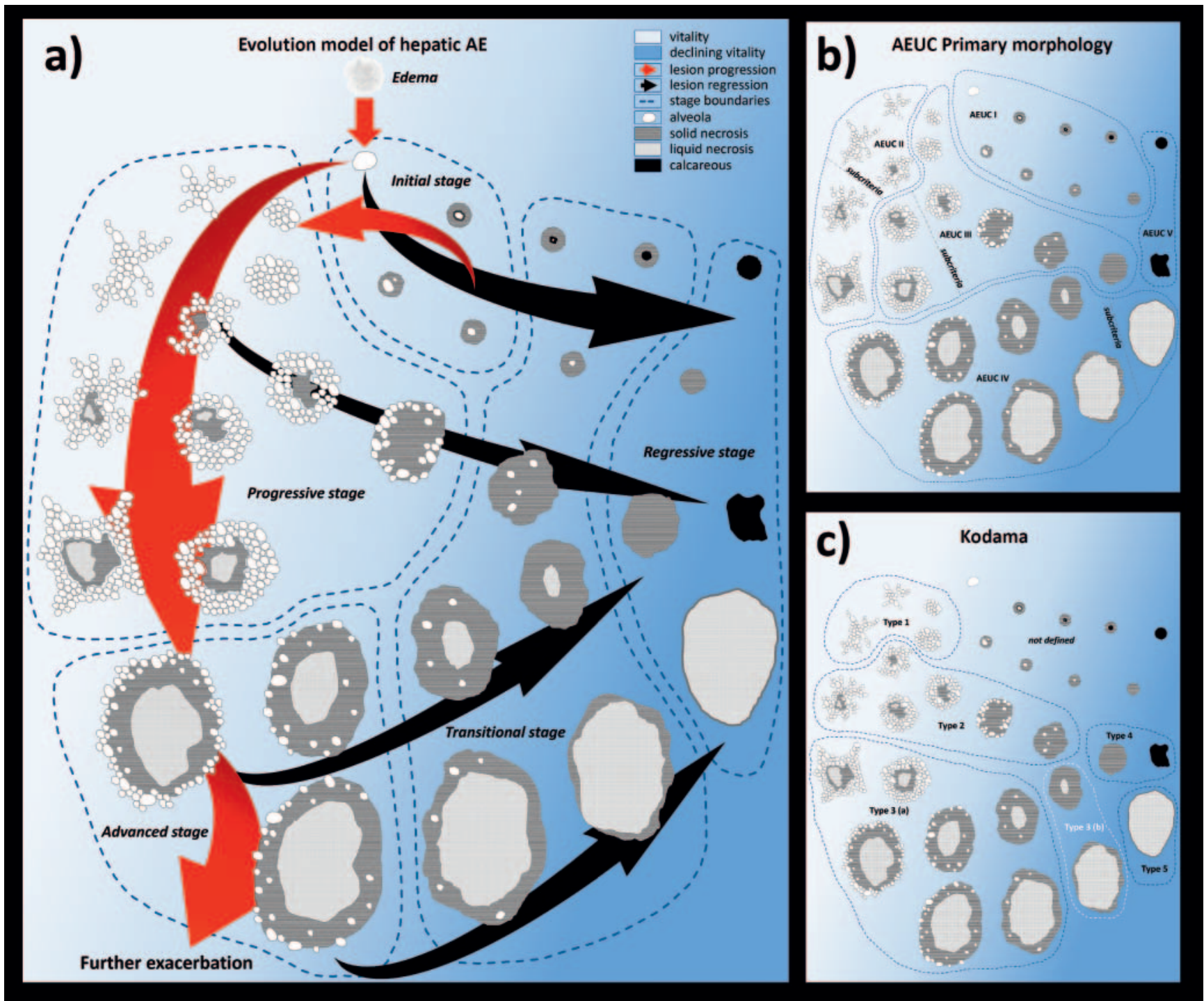

- Fig. 5 a Classification scheme of an intermodal approach to the evolution of hepatic AE lesions. Five stages are distinguished: "initial stage", "progressive stage", "advanced stage", "transitional stage", "regressive stage". Further parameters that have value in the assessment of AE lesions are noted in the main text. In particular, calcifications (AEUC pattern of calcification and grade of calcification) that are well assessable by CT contribute complementarily to the overall assessment, with a potential increase over time and under therapy, as well as knowing that the "feathery calcifications" are a sign of the highest inflammatory activity. b Assignment of the schematically presented lesions of the intermodal classification scheme to the AEUC primary morphologies of CT (including subcriteria for AEUC II-IV). c Assignment of the different schematically presented lesions of the intermodal classification scheme to the Kodama types of MRI (including the latest proposal with distinction in Kodama 3a and 3b).

documented by follow-up MRI examinations and within two years the finding resulted in two FDG-PET-positive AEUC-III lesions on CT. A small initial alveolar lesion, which in contrast to a normal small liver cyst has surrounding edema on diffusion-weighted (DWI) MRI and T2w, can be identified even prior to the formation of the alveolus based on the local edema. Edema is apparently the first visible morphological sign of a new hepatic AE infection ( Fig. 5a, 6).

It is problematic that such small lesions are not included in the primary T2w-based MRI Kodama classification [17] and can easily be misinterpreted as small simple cysts or even overlooked due to their minimal size [25, 26, 32]. The hypointense T1w signal in addition to $\mathrm{T} 2 \mathrm{w}$ can sometimes help to better delimit initial lesions with only a small central alveolus. In contrast on CT, additionally the marginal solid hypodense necrosis can be visualized more clearly and in combination with an even smaller central alveolus - if the latter is present - such lesions as a whole present as small, but well delimitable hypodense manifestations in this modality [25]. US can visualize the internal structures of such small lesions with the highest resolution but has problems with the precise visualization of large and calcified AE lesions (hailstorm pattern) $[18,20]$ ( $\triangleright$ Fig. 7).

Since there were only few points differentiating the primary morphologies "diffuse infiltrating" (AEUC II) and "primarily circumscribed tumor-like" (AEUC III) from one another in most precursor studies, those two forms of manifestation can be seen as 

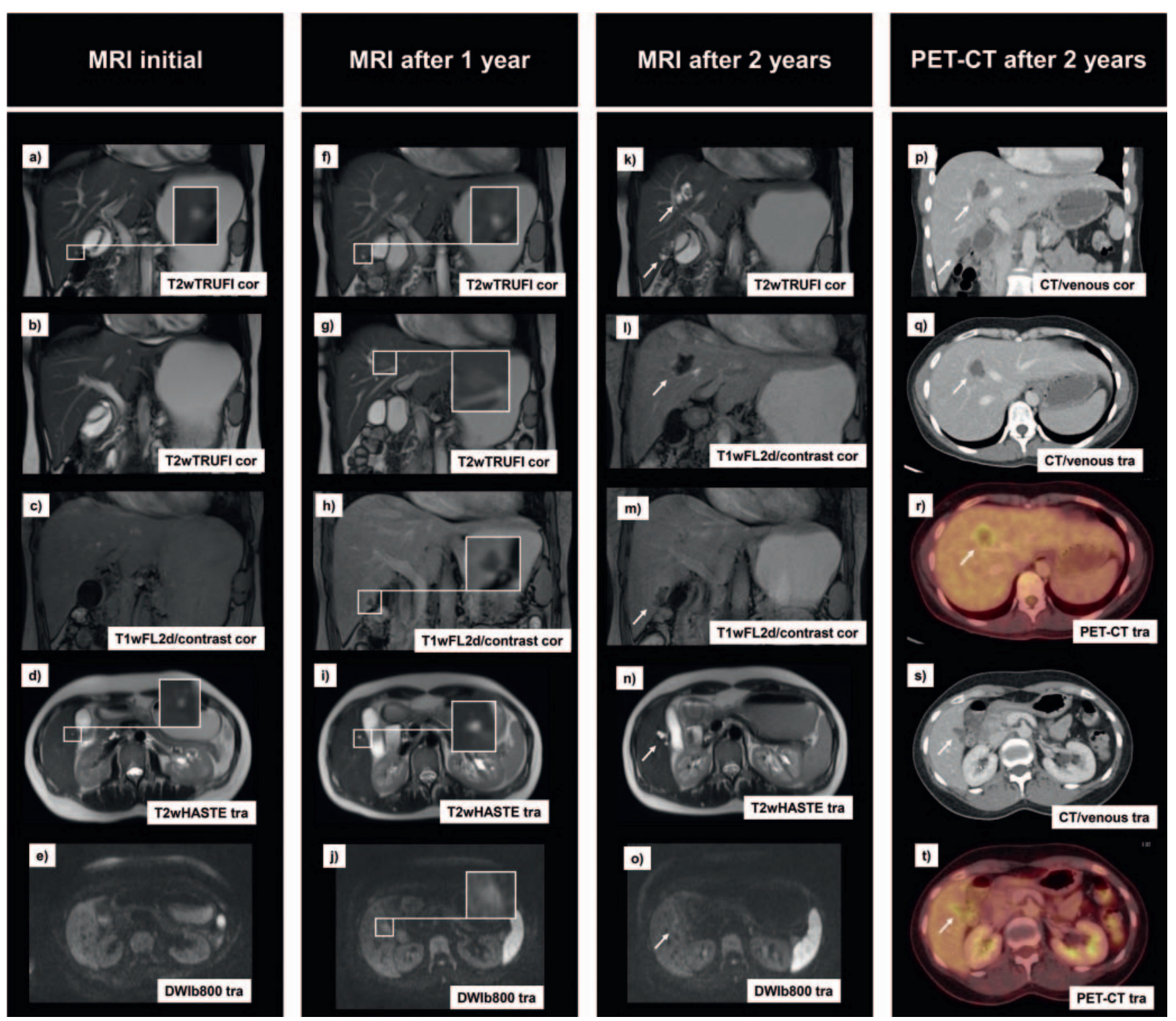

- Fig. 6 Directly traceable initial AE lesion evolution on MRI and PET-CT over the course of two years. The initial MRI examination shows a tiny cyst (alveolus) located in liver segment 5 that is hyperintense in T2w a, d. No surrounding contrast enhancement can be detected in this area $\mathbf{c}$ and the presence of local edema is not yet clear $\mathbf{d}$, e. No alteration can yet be detected in the area of a later lesion in segment $8 \mathbf{b}$. In the MRI examination performed one year later, the still tiny cyst (alveolus) in liver segment 5 has grown a little as can be seen in T2w $\mathbf{f}$, $\mathbf{i}$ and can be delimited even more in T1 $\mathbf{w}$ as hypointense, now with subtle surrounding contrast enhancement $\mathbf{h}$. At this point, T2w indicates flat ambient edema $\mathbf{i}$, which is more clearly shown in DWI $\mathbf{j}$. A further instance of slight edema in T2w recently occurred in segment $8 \mathbf{g}$, which had not been seen in this location in the previous examination $\mathbf{b}$. A microcyst cannot yet be delimited in the area of the newly occurring edema $\mathbf{g}$. In the MRI examination two years later in both locations of segment 5 and 8, already bigger, AE-typical lesions can now be delineated with microcysts and starting solid portions that most closely are to be classified as Kodama type 2 lesions $\mathbf{k}, \mathbf{n}$. Both lesions show shallow surrounding contrast enhancement I, $\mathbf{m}$. The surrounding edema (shown for the lesion in segment 5) has already mostly diminished compared to the preliminary examination $\mathbf{n}, \mathbf{o}$. A complementary PET-CT examination performed two years after initial MRI clearly demonstrates the two findings in segments 5 and 8 that already correspond to AEUC III p, $\mathbf{q}$, s. Some peripherally located microcysts can be recognized as small hypodense structures. Both lesions show increased surrounding inflammatory PET activity $\mathbf{r}, \mathbf{t}$.

different morphological expressions of early and intermediate lesion progression with a possible change between the two morphologies [27].

Current studies show that various lesion types can become a "primarily calcified" AEUC V lesion in the "regressive stage" due to a decrease in size and increasing calcification. A direct transition from small AEUC I lesions - spontaneous or during benzimi- dazole therapy - to correspondingly small, calcified lesions is certainly most common [22, 27].

A study comparing PET activity and MRI morphology according to Kodama showed that a reduction of the inflammatory response of the lesions can be attributed to the loss of the alveoli [29]. Based on this, solid and cystic AE lesions without alveoli (Kodama types 4 and 5) were also described as types of regression [31]. 

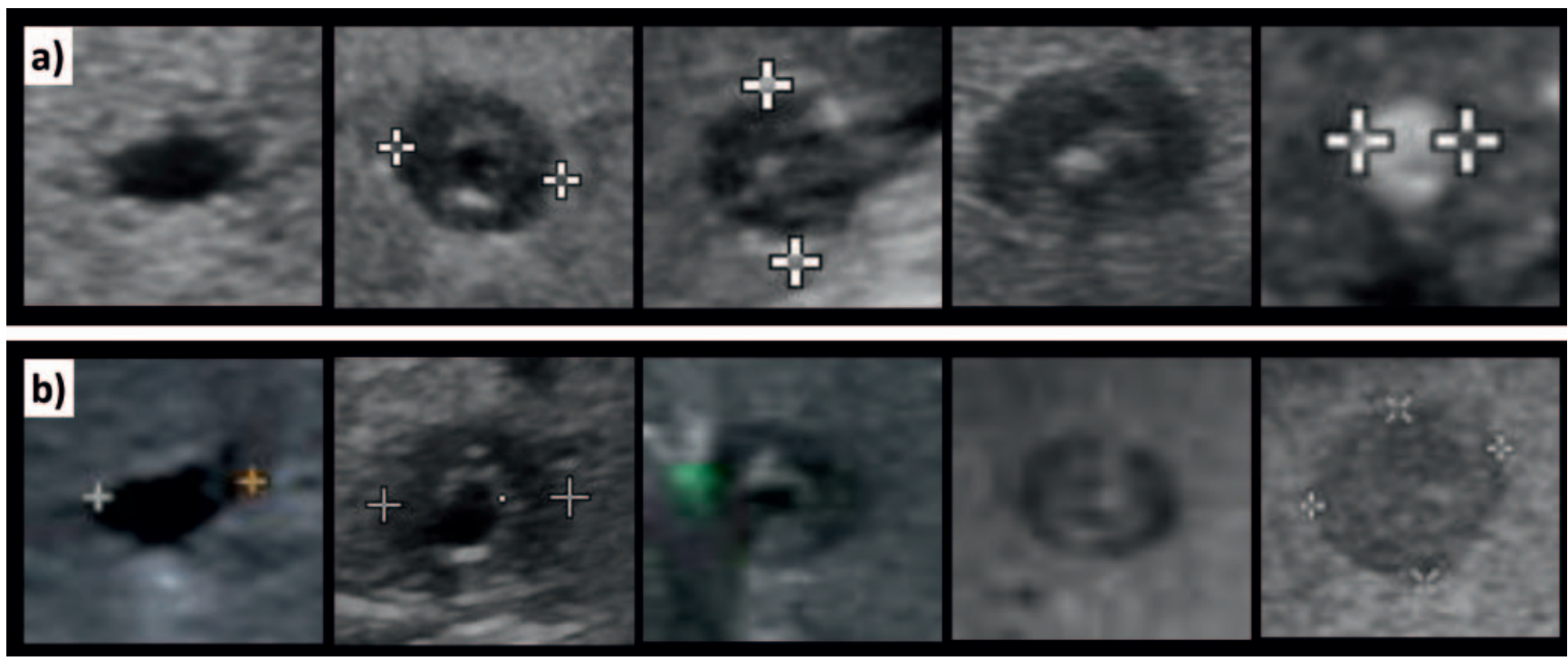

- Fig. 7 Ultrasound can visualize the internal structures of small initial lesions with the highest resolution. The initial alveolus carrying the lamellar body may at first be surrounded by isolating solid necrosis a, b left side. In addition to exacerbation through the active alveolus, initial observations suggest that small lesions may also pass into regressive forms, either via increase in centrifugally spreading calcification a or by complete solid necrosis $\mathbf{b}$, in each case at the cost of the central alveolus.

Thus, in addition to complete calcification as a form of regression, further types of regression in the form of completely solid or liquid necrotizing lesions are possible. In this context, solid lesions tend toward greater calcification than primarily liquid lesions [33]. In regard to CT, not only AEUC I but occasionally also AEUC III and IV lesions in the case of progressive decimation of alveoli with an increase in solid or liquid necroses can undergo an additional "transitional stage" to regressive lesions. Therefore, such a "transitional stage", which can extend beyond the boundaries of various primary lesion morphologies, was included in the intermodal evolution concept ( $\vee$ Fig. 5a). In the case of lesions in the "transitional stage", reactivation and exacerbation seem increasingly unlikely. In contrast, in the preceding "progressive stage" and in the "advanced stage", the site of disease activity is still the margins of the lesion, allowing further exacerbation. Therefore, MRI with visualization of alveoli offers important information for the differentiated evaluation of such lesions [17, 29].

Like the international epidemiological CT comparison studies mentioned in the introduction [23, 24], two corresponding MRI studies based on the Kodama classification with 200 cases each support a categorization of AE lesions within the intermodal classification scheme of evolution of hepatic AE lesions shown in - Fig. 5a [30, 31]. Accordingly, Kodama types 1 and 2 are used to classify early and progressive lesions, Kodama type 3 advanced lesions, and Kodama types 4 and 5 degenerative lesions [30, 31, 33]. A differentiation between Kodama $3 \mathrm{a}$ and $3 \mathrm{~b}$ depending on the presence of alveoli is proposed [31]. The assignment of the schematically presented lesions of the intermodal evolution model to both the AEUC primary morphologies for CT and to the MRI
Kodama types is shown in $\mathbf{F i g . 5 b}$, c. Comparison to $\mathbf{F i g . 5 a}$ shows that due to method-dependent overlapping of the individual systems the assignment of an AE lesion to a certain stage can be performed more precisely when the image information of the classifications of both modalities are combined. The morphologies of the EMUC-US can also be transferred to the evolution scheme, e. g., "metastasis-like", "pseudocystic", or "ossification" pattern. However, larger comparative studies on US are not currently available.

In addition to the schematic drawing of lesion morphology in - Fig. 5a supplementary parameters that are important for the evaluation of AE lesions are the pattern and degree of calcification $[22,26,28]$, the possible subtle surrounding contrast enhancement with the typical lack of contrast enhancement of the actual lesion, and finally the peripheral PET activity and diffusion restriction [15, 26, 33-35]. Immunology, histology [27, 36-38], and serology $[26,39,40]$ are further pillars of the diagnosis. The currently available results of such parameters have been applied to the development of the evolution model. The presented scheme provides a systematic foundation for further studies on the indicated parameters, particularly in light of the described necessity of unimodal as well as intermodal assessment of hepatic AE lesions.

The classification systems presented here allow standardized diagnosis adapted to the development stages of AE lesions. Prospective observation of untreated $\mathrm{AE}$ cases to establish such systems would not be ethical. However, on the presented basis prospective studies on a possible influence of lesion stages on treatment and the prognosis of AE particularly with respect to non-operable cases should be performed. 


\section{Conflict of Interest}

The authors declare that they have no conflict of interest.

\section{Acknowledgments}

\section{Echinococcosis Study Group Ulm:}

Thomas FE Barth, Sven Baumann, Ambros Beer, Meinrad Beer, Johannes Bloehdorn, Nina Eberhardt, Iris Fischer, Tilmann Graeter, Johannes Grimm, Beate Gruener, Doris Henne-Bruns, Andreas Hillenbrand, Tanja Kaltenbach, Wolfgang Kratzer, Patrycja Schlingeloff, Julian Schmidberger, Rong Shi

\section{References}

[1] Kern P, Bardonnet K, Renner E et al. European echinococcosis registry: human alveolar echinococcosis, Europe, 1982-2000. Emerg Infect Dis 2003; 9: 343-349. doi:10.3201/eid0903.020341

[2] Moro P, Schantz PM. Echinococcosis: a review. Int J Infect Dis 2009; 13 : 125-133. doi:10.1016/j.ijid.2008.03.037

[3] Baumann S, Shi R, Liu W et al. Worldwide literature on epidemiology of human alveolar echinococcosis: a systematic review of research published in the twenty-first century. Infection 2019; 47: 703-727. doi:10.1007/s15010-019-01325-2

[4] Romig T, Kratzer W, Kimmig P et al. An epidemiologic survey of human alveolar echinococcosis in southwestern Germany. Römerstein Study Group. Am J Trop Med Hyg 1999; 61: 566-573. doi:10.4269/ ajtmh.1999.61.566

[5] Torgerson PR, Keller K, Magnotta M et al. The global burden of alveolar echinococcosis. PLoS Negl Trop Dis 2010; 4: e722. doi:10.1371/journal. pntd. 0000722

[6] Deplazes P, Rinaldi L, Alvarez Rojas CA et al. Global Distribution of Alveolar and Cystic Echinococcosis. Adv Parasitol 2017; 95: 315-493. doi:10.1016/bs.apar.2016.11.001

[7] Zhang W, Zhang Z, Wu W et al. Epidemiology and control of echinococcosis in central Asia, with particular reference to the People's Republic of China. Acta Trop 2015; 141: 235-243. doi:10.1016/j.actatropica.2014.03.014

[8] Craig PS. Echinococcosis Working Group in China. Epidemiology of human alveolar echinococcosis in China. Parasitol Int 2006; 55: 221225. doi:10.1016/j.parint.2005.11.034

[9] Conraths F], Probst C, Possenti A et al. Potenzial risk factors associated with human alveolar echinococcosis: Systematic review and meta-analysis. PLoS Negl Trop Dis 2017; 11: e0005801. doi:10.1371/journal.pntd.0005801

[10] Ammann RW, Eckert J. Cestodes. Echinococcus. Gastroenterol Clin North Am 1996; 25: 655-689. doi:10.1016/s0889-8553(05)70268-5

[11] Eckert J, Deplazes P. Biological, epidemiological, and clinical aspects of echinococcosis, a zoonosis of increasing concern. Clin Microbiol Rev 2004; 17: 107-135. doi:10.1128/cmr.17.1.107-135.2004

[12] Hillenbrand A, Gruener B, Kratzer W et al. Impact of Safe Distance on Long-Term Outcome After Surgical Therapy of Alveolar Echinococcosis. World J Surg 2017; 41: 1012-1018. doi:10.1007/s00268-016-3813-6

[13] Graeter T, Ehing F, Oeztuerk $S$ et al. Hepatobiliary complications of alveolar echinococcosis: A long-term follow-up study. World J Gastroenterol 2015; 21: 4925-4932. doi:10.3748/wjg.v21.i16.4925

[14] Brunetti E, Kern P, Vuitton DA. Writing Panel for the WHO-IWGE. Expert consensus for the diagnosis and treatment of cystic and alveolar echinococcosis in humans. Acta Trop 2010; 114: 1-16. doi:10.1016/ j.actatropica.2009.11.001

[15] Kaltenbach TE, Graeter T, Mason RA et al. Determination of vitality of liver lesions by alveolar echinococcosis. Comparison of parametric contrast enhanced ultrasound (SonoVue ${ }^{\circledR}$ ) with quantified 18F-FDG-PET-CT. Nuklearmedizin 2015; 54: 43-49. doi:10.3413/Nukmed-0670-14-05
[16] Ricken F], Nell ], Grüner B et al. Albendazole increases the inflammatory response and the amount of Em2-positive small particles of Echinococcus multilocularis (spems) in human hepatic alveolar echinococcosis lesions. PLoS Negl Trop Dis 2017; 11: e0005636. doi:10.1371/journal. pntd.0005636

[17] Kodama Y, Fujita N, Shimizu T et al. Alveolar echinococcosis: MR findings in the liver. Radiology 2003; 228: 172-177. doi:10.1148/ radiol.2281020323

[18] Kratzer W, Gruener B, Kaltenbach TE et al. Proposal of an ultrasonographic classification for hepatic alveolar echinococcosis: Echinococcosis multilocularis Ulm classification-ultrasound. World ] Gastroenterol 2015; 21: 12392-12402. doi:10.3748/wjg.v21.i43

[19] Graeter T, Kratzer W, Oeztuerk $S$ et al. Proposal of a computed tomography classification for hepatic alveolar echinococcosis. World J Gastroenterol 2016; 22: 3621-3631. doi:10.3748/wjg.v22.i13.3621

[20] Grüner B, Schmidberger ], Drews $O$ et al. Imaging in alveolar echinococcosis (AE): Comparison of Echinococcus multilocularis classification for computed-tomography (EMUC-CT) and ultrasonography (EMUC-US). Radiology of Infectious Diseases 2017; 4: 70-77. doi:10.1016| j.jrid.2017.05.001

[21] Kern P, Wen H, Sato N et al. WHO classification of alveolar echinococcosis: principles and application. Parasitol Int 2006 (Suppl. 55): S283S287. doi:10.1016/j.parint.2005.11.041

[22] Graeter T, Shi R, Bao HH et al. Follow-up in hepatic alveolar echinococcosis under benzimidazole therapy using computed tomography. Chin Med J (Engl) 2020; 133: 1507-1509. doi:10.1097| CM9.0000000000000874

[23] Graeter T, Bao H, Delabrousse E et al. Hepatic alveolar echinococcosis: Comparative computed tomography study between two Chinese and two European centres. Chin Med J (Engl) 2020; 19: e00082. doi:10.1016/j.fawpar.2020.e00082

[24] Graeter T, Bao HH, Shi R. et al. Evaluation of intrahepatic manifestation and distant extrahepatic disease in alveolar echinococcosis. World J Gastroenterol 2020; 26: 4302-4315. doi:10.3748/wjg.v26.i29.4302

[25] Engler A, Shi R, Beer $M$ et al. Simple liver cysts and cystoid lesions in hepatic alveolar echinococcosis: A retrospective cohort study with Hounsfield analysis. Parasite 2019; 26: 54. doi:10.1051/parasite/ 2019057

[26] Graeter T, Eberhardt N, Shi R et al. Hepatic alveolar echinococcosis: Correlation between computed tomography morphology and inflammatory activity in positron emission tomography. Sci Rep 2020; 10 : 11808. doi:10.1038/s41598-020-68624-9

[27] Grimm J, Beck A, Nell J et al. Combining Computed Tomography and Histology Leads to an Evolutionary Concept of Hepatic Alveolar Echinococcosis. Pathogens 2020; 9: 634. doi:10.3390/pathogens9080634

[28] Brumpt E, Blagosklonov O, Calame P et al. AE hepatic lesions: correlation between calcifications at CT and FDG-PET/CT metabolic activity. Infection 2019; 47: 955-960. doi:10.1007/s15010-019-01328-z

[29] Azizi A, Blagosklonov O, Lounis A et al. Alveolar echinococcosis: correlation between hepatic MRI findings and FDG-PET/CT metabolic activity. Abdominal Imaging 2015; 40: 56-63. doi:10.1007/s00261-014-0183-0

[30] Graeter T, Shi R, Bao HH et al. Intrahepatic manifestation and distant extrahepatic disease in alveolar echinococcosis: a multicenter cohort study. Acta Radiol 2020. doi:10.1177/0284185120951958

[31] Brumpt W, Liu W, Graeter T et al. Kodama-XUUB: an informative classification for alveolar echinococcosis hepatic lesions at MRI. Parasite. In press.

[32] Aoki T, Hagiwara M, Yabuki H et al. Unique MRI findings for differentiation of an early stage of hepatic alveolar echinococcosis. BMJ Case Rep 2015; 2015. doi:10.1136/bcr-2014-208123

[33] Becce F, Pomoni A, Uldry E et al. Alveolar echinococcosis of the liver: diffusion-weighted MRI findings and potential role in lesion characterisation. Eur J Radiol 2014; 83: 625-631. doi:10.1016/j.ejrad.2013.12.025 
[34] Liu W, Delabrousse É, Blagosklonov O et al. Innovation in hepatic alveolar echinococcosis imaging: best use of old tools, and necessary evaluation of new ones. Parasite 2014; 21: 74. doi:10.1051/parasite/2014072

[35] Parry AH, Wani AH, Feroz I. The spectrum of multimodality imaging findings in hepatic alveolar echinococcosis and the potential role of diffusion-weighted imaging in its characterisation. Pol J Radiol 2020; 85: e613-e623. doi:10.5114/pjr.2020.101015

[36] Tappe D, Zidowitz S, Demmer P et al. Three-dimensional reconstruction of Echinococcus multilocularis larval growth in human hepatic tissue reveals complex growth patterns. Am J Trop Med Hyg 2010; 82: 126127. doi:10.4269/ajtmh.2010.09-0462

[37] Reinehr M, Micheloud C, Grimm F et al. Pathology of Echinococcosis: A Morphologic and Immunohistochemical Study on 138 Specimens With Focus on the Differential Diagnosis Between Cystic and Alveolar Echinococcosis. Am J Surg Pathol 2020; 44: 43-54. doi:10.1097|

PAS.0000000000001374
[38] Barth TF, Herrmann TS, Tappe D et al. Sensitive and specific immunohistochemical diagnosis of human alveolar echinococcosis with the monoclonal antibody Em2G11. PLoS Negl Trop Dis 2012; 6: e1877. doi:10.1371/journal.pntd.0001877

[39] Deplazes P, Gottstein B. A monoclonal antibody against Echinococcus multilocularis Em2 antigen. Parasitology 1991; 103: 41-49. doi:10.1017/s0031182000059278

[40] Gottstein B, Lachenmayer A, Beldi G et al. Diagnostic and follow-up performance of serological tests for different forms/courses of alveolar echinococcosis. Food Waterborne Parasitol 2019; 16: e00055. doi:10.1016/j.fawpar.2019.e00055 